\title{
Twin study on cup/disc ratio of the optic nerve head
}

\author{
Jaakko M Teikari, Juhani P Airaksinen
}

\begin{abstract}
Seventeen healthy twin pairs (10 monozygotic and seven dizygotic) from the Finnish Twin Cohort Study were examined to study the impact of heredity $v$ environment in the determination of cup-to-disc area ratio. These twins were free from any known eye disease. The cup/ disc ratio was determined using stereo photography and a computer assisted analysis technique. The zygosity of all twin pairs was confirmed with the DNA 'fingerprint' technique. The intrapair correlations were high among monozygotic pairs compared with those among dizygotic twin pairs. The difference of cup/disc area ratios between the right eyes of members of monozygotic twin pairs was statistically significantly smaller than that of dizygotic twin pairs $(p<0.001)$. The same was true for left eyes $(\mathbf{p}<0.01)$. This result confirms a genetic determination in cup/disc area ratio in normal eyes.
\end{abstract}

A previous study of Armaly has shown that the cup/disc ratio of the optic nerve head is genetically determined. ${ }^{1}$ He examined parent-offspring and sibling-sibling pairs and found that the cup/disc ratios of these first degree relatives were similar. $\mathrm{He}$ found no such similarity between husbandwife pairs. The problem with such a genealogical model is that both genetic and environmental factors could cause sib-sib and parent-offspring similarities. It seems obvious that the effects of genes and environment need to be separated to confirm this important finding. One way to separate these two sources of variation is the twin study method. Here the simultaneous occurrence of a disease or trait in both members of a twin pair is called concordance. When the disease affects only one member of a pair it is called discordance. The twin method compares the concordances among monozygotic twins with that of dizygotic twins. In a monogenetically transmitted disease the monozygotic twins should be $100 \%$ concordant and the dizygotic twins $50 \%$ concordant. When a continuous trait is under study the within-pair correlations among monozygotic twin pairs are compared with those among dizygotic pairs. The excess correlation among monozygotic pairs is attributed to genetic effects to the trait. We used the nationwide Finnish Twin Cohort Study to apply the twin method to cup/disc ratio of the optic nerve head.

University of Oulu, Department of

Ophthalmology, Oulu, Finland

J P Airaksinen

Correspondence to:

Jaakko Teikari, University of

Helsinki, Department of

Ophthalmology,

Ophthalmology,
Haartmaninkatu 4,00290

Helsinki, Finland.

Accepted for publication

22 August 1991

\section{Material and methods}

The pairs were selected randomly from one age stratum (34-35 years) of a nationwide twin registry called the Finnish Twin Cohort Study (FTCS). The compilation, zygosity determination, and description of the cohort have been presented in previous publications. ${ }^{2-6}$ This twin
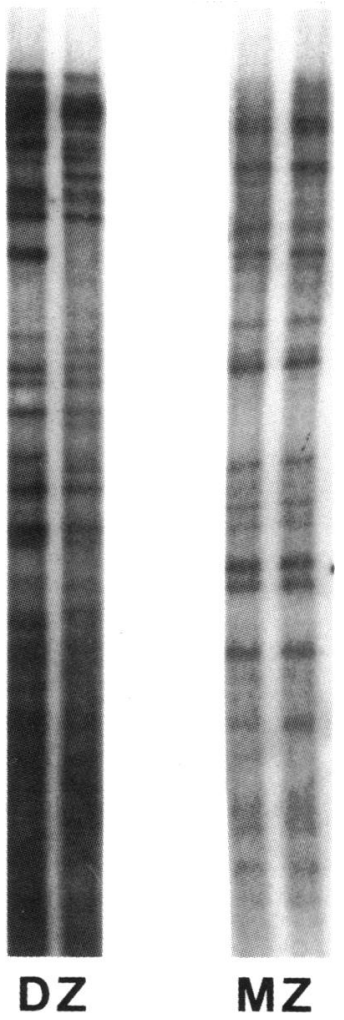

Figure 1 DNA 'fingerprints' of a monozygotic $(M Z)$ and dizygotic $(D Z)$ twin pair showing the exact similarity of bands in the monozygotic pair.

cohort comprises all twin pairs in Finland and currently includes 16000 twin pairs both monozygotic and same-sexed dizygotic. Twenty pairs were invited to the study (compliance $90 \%$ ). A complete clinical eye examination was performed including visual acuity, objective refraction, slit-lamp examination, applanation tonometry, indirect ophthalmoscopy, and ultrasound measurement of the axial length and anterior chamber depth. The optic nerve head was photographed with a Canon fundus camera on black and white film. The stereo base was created using lateral shifting technique. Optic disc, cup, and neuroretinal rim areas as well as cup/disc area ratios were digitised manually from the stereophotographs with a computer assisted technique. The software incorporates algorithms to take into account image magnification/minification induced by axial ametropias. ${ }^{7}$ As a result actual optic disc and optic cup areas were obtained in square millimetres. The examiner of the stereo photographs was unaware of the identity of the particular eye under study. The zygosity has been determined for the whole cohort by a questionnaire method. ${ }^{6}$ It was verified in this particular sample by the DNA 'fingerprint' technique. ${ }^{8}$ Figure 1 shows an example of one monozygotic and one dizygotic pair and their DNA 'fingerprints'. The bands for a mono- 
Table 1 Mean disc areas, cup areas, and cup/disc ratios by zygosity and laterality (value of member A/value of member $B$ )

\begin{tabular}{lllllllll}
\hline & Disc area & $S D$ & Cuparea & $S D$ & Rimarea & $S D$ & Cup/disc ratio & $S D$ \\
\hline MZ & & & & & & & & \\
Right eyes & $2 \cdot 23 / 2 \cdot 13$ & $0 \cdot 39 / 0 \cdot 47$ & $0 \cdot 71 / 0 \cdot 59$ & $0 \cdot 40 / 0 \cdot 43$ & $1 \cdot 52 / 1 \cdot 54$ & $0 \cdot 20 / 0 \cdot 32$ & $0 \cdot 30 / 0 \cdot 26$ & $0 \cdot 13 / 0 \cdot 15$ \\
Left eyes & $2 \cdot 14 / 2 \cdot 14$ & $0 \cdot 37 / 0 \cdot 34$ & $0 \cdot 61 / 0 \cdot 55$ & $0 \cdot 39 / 0 \cdot 40$ & $1 \cdot 52 / 1 \cdot 59$ & $0 \cdot 36 / 0 \cdot 29$ & $0 \cdot 28 / 0 \cdot 25$ & $0 \cdot 16 / 0 \cdot 14$ \\
$D Z$ & & & & & & & \\
Right eyes & $2 \cdot 19 / 2 \cdot 11$ & $0 \cdot 46 / 0 \cdot 31$ & $0 \cdot 38 / 0 \cdot 43$ & $0 \cdot 39 / 0 \cdot 28$ & $1 \cdot 80 / 1 \cdot 68$ & $0 \cdot 23 / 0 \cdot 24$ & $0 \cdot 16 / 0 \cdot 19$ & $0 \cdot 14 / 0 \cdot 12$ \\
Left eyes & $2 \cdot 03 / 2 \cdot 06$ & $0 \cdot 38 / 0 \cdot 32$ & $0 \cdot 37 / 0 \cdot 40$ & $0 \cdot 26 / 0 \cdot 34$ & $1 \cdot 66 / 1 \cdot 65$ & $0 \cdot 29 / 0 \cdot 18$ & $0 \cdot 18 / 0 \cdot 18$ & $0 \cdot 10 / 0 \cdot 13$ \\
\hline
\end{tabular}

zygotic twin pair are exactly alike while the dizygotic pairs differ in several bands.

One pair from the original 20 pairs invited was discarded because the second member of the pair was unable to attend the examination because of estimated delivery in 2 weeks. Another pair was discarded from this analysis because of missing photographs of the second member of the pair. One pair was discarded because one member of the pair did not attend the clinical examination. One eye of a dizygotic twin was discarded from the calculations because of a non-alternating strabismus and amblyopia. All eyes in the calculations had normal visual acuity and no previous or present eye disease.

\section{Results}

The mean disc areas, cup areas, and cup/disc ratios for both zygosity classes and zygosities are given in Table 1. In Figure 2 the distributions of the cup/disc ratios are given. From the figure it appears that the cup/disc ratios are not normally distributed. Because of this a non-parametric statistic was chosen in the calculations of correlation (Spearman rank correlation). The correlations in cup/disc ratios between right and left eyes in the same individual showed high correlations in both $\mathrm{MZ}$ and $\mathrm{DZ}$ twin pairs (Table 2). The correlations between right-right and left-left eyes between two members of a monozygotic twin pair were fourfold compared with the same correlations among two members of a dizygotic twin pair (Table 3 ). The mean absolute differences in cup/disc area ratio between right $(0.062)$ and left $(0.037)$ eyes of monozygotic twins

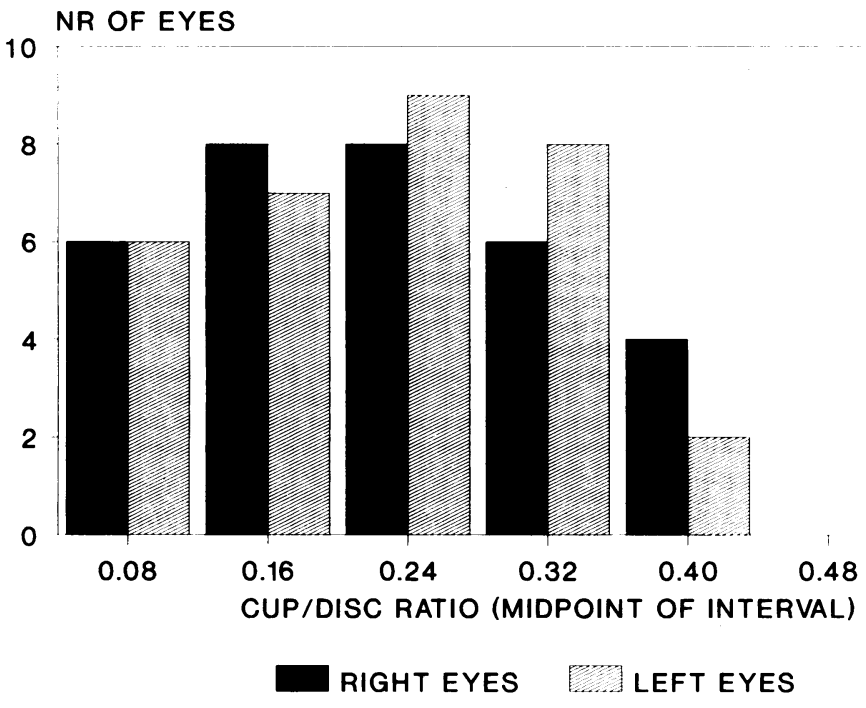

Figure 2 Distribution of cup/disc area ratios in this sample of 17 twin pairs. were statistically significantly lower than right $(0 \cdot 181)$ and left $(0 \cdot 132)$ eyes of dizygotic twins $(p<0.001$ right eyes, $p<0.005$ left eyes, Wilcoxon two-sample test). In Figure 3 an example of an optic nerve head of a monozygotic and a dizygotic pair (right eyes of twin sisters) is shown. The cup/disc ratios are remarkably similar between the two members of the monozygotic pair while there is a substantial difference in the dizygotic twin pair (right eyes of twin sisters).

The result of this study supports the theory of a strong genetic determination of cup/disc ratio in normal eyes.

\section{Discussion}

The present material confirms the genetic nature of determination of the cup/disc area ratio in a normal eye.

The disc areas were similar in both monozygotic and dizygotic pairs but the cup areas were smaller among dizygotic twin pairs both in right and left eyes. The smaller mean cup sizes and cup/disc ratios in dizygotic twin pairs could decrease the differences among dizygotic twin pairs thus resulting in an underestimation of heredity in this sample and hereby increasing the validity of our conclusion.

Table 2 Intra-individual correlations in cup/disc ratios between right and left eyes of $10 \mathrm{MZ}$ and seven $D Z$ twin pairs (Spearman rank correlation)

\begin{tabular}{lll}
\hline$M Z$ & A ODX & \\
A OSIN & 0.90 & B ODX \\
B OSIN & & 0.82 \\
$D Z$ & A ODX & B ODX \\
A OSIN & 0.64 & 0.80 \\
B OSIN & & \\
\hline
\end{tabular}

$A=$ first member of a twin pair.

$B=$ second member of a twin pair.

$\mathrm{ODX}=$ right eye.

ODX $=$ right eye
OSIN $=$ left eye.

Table 3 Intrapair correlations of cup/disc ratios of 10 monozygotic and seven dizygotic twin pairs (Spearman rank correlation). (The correlations are shown separately for right eyes and left eyes of twin pairs

$M Z$

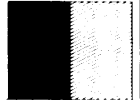

B ODX

B OSIN

DZ

0.56

B ODX

B OSIN

A ODX

A OSIN

0.98

A OSIN

A ODX

$0 \cdot 14$ 
Monozygotic pair
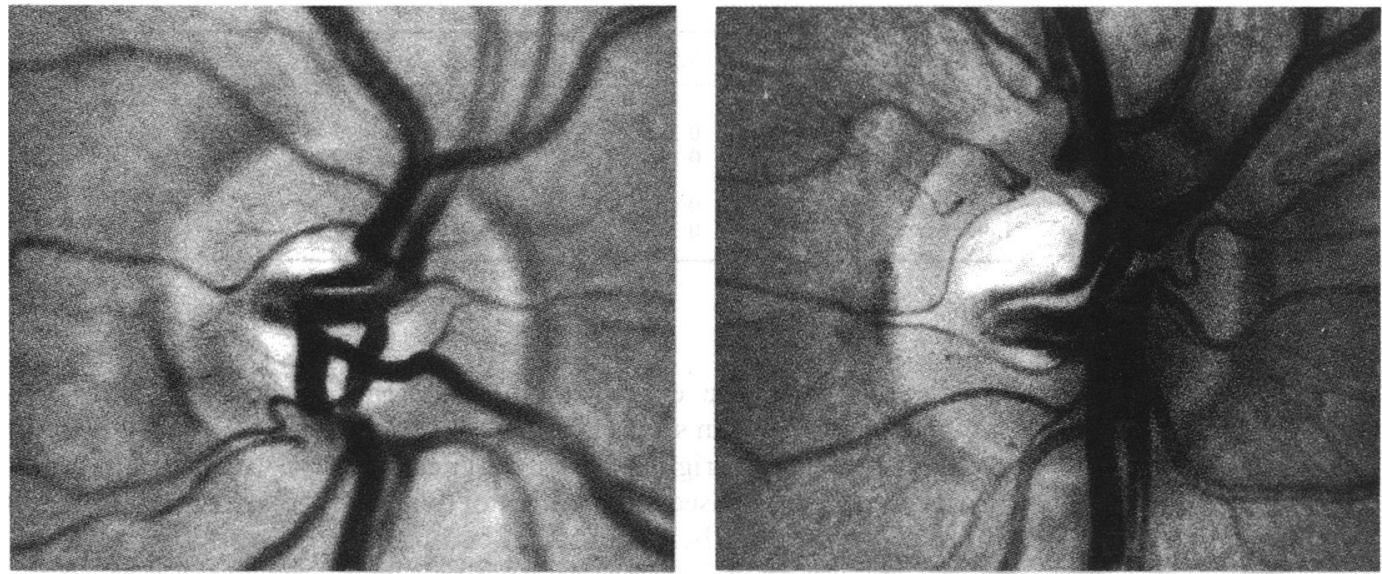

Dizygotic pair
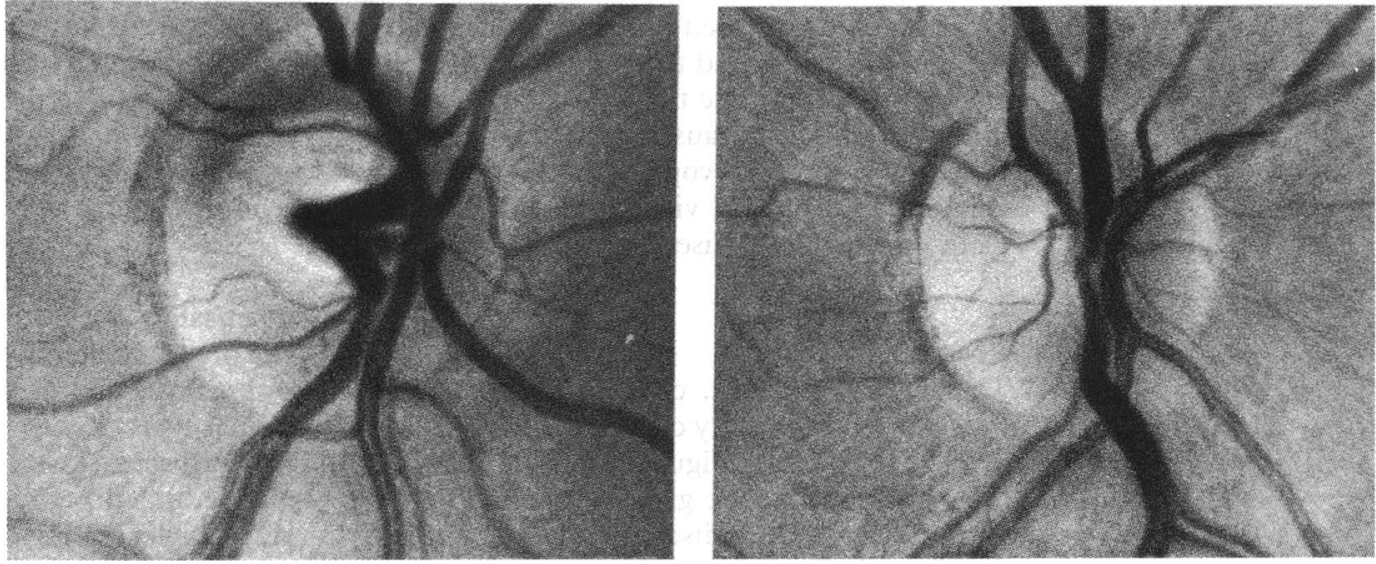

Figure 3 Example of the optic nerve head of a monozygotic and a dizygotic twin pair. The upper two pictures represent right eyes of a female monozygotic pair showing a significant similarity of the cup/disc ratio. The lower two pictures represent right eyes of a female dizygotic pair showing a significant dissimilarity in the cup/disc ratio.

The distribution of cup/disc area ratios in this sample was skewed. Lower values were overrepresented. This is in accordance with previous findings by Armaly.' The high correlation between two eyes of any given individual was confirmed in the present sample. A previous study by Armaly showed that the cup/disc ratio differed less than 0.1 in $92 \%$ of 1098 subjects and less than 0.2 in $99 \%$ of subjects. In large samples of healthy individuals the cup/disc ratio of one eye alone or mean of both eyes can be used without causing bias due to intraperson variation between the two eyes.

The high heritability of the cup/disc ratio in normal eyes has important implications. It has been shown previously by Armaly that the cup/ disc ratio of first degree relatives of glaucoma patients does not show a different distribution from that in the general population. ${ }^{1}$ This might imply that while the cupping of the optic nerve head itself is genetic in origin that of glaucomatous cupping is affected by environmental factors or perhaps a gene-environment interaction.

The liability to glaucoma might still be controlled genetically in part. The heritability of determination of the cup/disc ratio in a healthy eye would bias the heritability estimates of glaucoma if the cup/disc ratio is used as a major criterion for diagnosis.

This study was supported by grants from Silma ja Kudospankkisaatio, Silmasaatio, and Suomen Kulttuurirahasto. From the Kimura Laboratory of Clinical Investigation, Beckman Vision Center.

1 Armaly MF. Genetic determination of cup/disc ratio of the optic nerve. Arch Ophthalmol 1967; 78: 35-43.

2 Kaprio J, Sarna S, Koskenvuo M, Rantasalo I. The Finnish Twin Registry: formation and compilation, questionnaire study, zygosity determination procedures and research program. Prog Clin Biol Res 1978; 24: 179-84.

3 Kaprio J, Koskenvuo M, Artimo M, Sarna S, Rantasalo I. The Finnish Twin Registry: baseline characteristics. Section Materials, methods, representativeness and results for variables special to twin studies. Publications of the Department of Public Health Science, University of Helsinki M47, ment of Public

4 Kaprio J, Sarna S, Koskenvuo M, Rantasalo I. Baseline characteristics of the Finnish Twin Registry. Section II. History of symptoms and illnesses, use of drugs, physical characteristics, smoking, alcohol and physical activity. Publications of the Department of Public Health Science M37, Helsinki, 1978.

5 Koskenvuo M, Langinvainio H, Kaprio J, Rantassalo I, Sarna S. The Finnish Twin Registry: baseline characteristics. Section III. Occupational and psychosocial factors. Publications of the Department of Public Health Science, University of Helsinki M49, Helsinki, 1979.

6 Sarna S, Kaprio J, Sistonen P, Koskenvuo M. Diagnosis of twin zygosity by mailed questionnaire. Hum Hered 1978; 28 : $241-54$.

7 Airaksinen PJ, Drance SM, Schultzer M. Neuroretinal rim area in early glaucoma. Am $\mathcal{F}$ Ophthalmol $1985 ; 99: 1-4$.

8 Helminen P, Enholm C, Lokki M-L, Jeffreys A, Peltonen L. Application of DNA 'fingerprints' to paternity determina tions. Lancet 1988; i: 574-6. 